$\mathrm{UCLA} / 95 / \mathrm{TEP} / 14$

hep-th/9505005

\title{
Topology on the lattice; 2d Yang-Mills theories with a theta term
}

\author{
Tamás G. Kovács円 and E.T. Tomboulis \\ Department of Physics UCLA, Los Angeles, California 90024-1547, U.S.A. \\ Zsolt Schram \\ Department of Theoretical Physics, Kossuth Lajos University, H-4010 Debrecen, \\ POBox 5, Hungary
}

\begin{abstract}
We study two-dimensional $\mathrm{U}(N)$ and $\mathrm{SU}(N)$ gauge theories with a topological term on arbitrary surfaces. Starting from a lattice formulation we derive the continuum limit of the action which turns out to be a generalisation of the heat kernel in the presence of a topological term. In the continuum limit we can reconstruct the topological information encoded in the theta term. In the topologically trivial cases the theta term gives only a trivial shift to the ground state energy but in the topologically nontrivial ones it remains to be coupled to the dynamics in the continuum. In particular for the $\mathrm{U}(N)$ gauge group on orientable surfaces it gives rise to a phase transition at $\theta=\pi$, similar to the ones observed in other models. Using the equivalence of $2 \mathrm{~d} \mathrm{QCD}$ and a $1 \mathrm{~d}$ fermion gas on a circle we rewrite our result in the fermionic language and show that the theta term can be also interpreted as an external magnetic field imposed on the fermions.
\end{abstract}

\footnotetext{
${ }^{1}$ e-mail: kovacs@physics.ucla.edu

${ }^{2}$ e-mail: tomboulis@physics.ucla.edu

3e-mail: schram@tigris.klte.hu
} 


\section{Introduction}

Recently there has been a renewed interest in studying lattice models with a topological term. This was motivated mostly by the hope that the long-standing strong CP problem can be solved [1]. Recall that in four dimensional QCD one can in principle add a $\mathrm{CP}$ violating term, the so called theta term to the conventional Yang-Mills action. In nature however, strong interactions seem to respect the CP symmetry, so the coupling constant of this term $(\theta)$ must be zero or at least very small. The question is why nature is forced to choose the special value $\theta=0$. Up to now Monte Carlo studies of this model have been very limited mostly due to the following two difficulties. At first the theta term in Euclidean space-time is purely imaginary which makes the Boltzmann weight of some states negative. It means that the Boltzmann weight cannot be interpreted as a probability measure. This difficulty, however, has been largely overcome by some new simulation techniques developed in the last five years [2]. The other problem arising in Monte Carlo simulations is related to the fact that the theta term is of topological nature. In four dimensional QCD on the sphere the theta term is proportional to the instanton number, an integer that does not change upon continuous deformations of the field configuration. On the lattice however, the notion of continuity is completely lost, or in other words continuity does not impose any restriction on the deformation of the degrees of freedom at different locations. As a result there are no disconnected instanton sectors and the very definition of the topological charge is ambiguous. A possible way of defining the topological charge is by picking a smooth "interpolating" field that is in some sense close to the given lattice configuration and assigning the topological charge of the interpolating field to the lattice configuration. This procedure is highly nontrivial and it is not clear what happens in the quantum continuum limit. An interesting question one can ask is whether the topological information encoded in the theta term is retained in the continuum limit.

In this paper we shall study this question in two-dimensional $\mathrm{U}(N)$ and $\mathrm{SU}(N)$ Yang-Mills theories defined on surfaces of different topologies. In this case the topo- 
logical term is proportional to the first Chern number of the underlying bundle. Our choice of the lattice theta term is the most naive, the only requirement it satisfies is that in the naive continuum limit it gives the Chern number. For $\mathrm{U}(N)$ bundles on orientable surfaces the first Chern number can take any integer value depending on the "twist" in the bundle. On the other hand it is identically zero for the $\mathrm{SU}(N)$ groups and also for $\mathrm{U}(N)$ on non-orientable surfaces. Even in the latter cases, the same "thetaterm" as for the nontrivial $\mathrm{U}(N)$ case, can be included in the lattice action. With our choice of the lattice theta term it does not vanish for any of the above discussed cases, it does not necessarily give an integer topological charge on the lattice, but it has the correct continuum behaviour. We shall show that the topological information encoded in the theta term can be completely recovered in the continuum limit. This means that in those cases where the Chern number is trivial, the theta term completely decouples from the dynamics, giving only a constant shift of the free energy. On the other hand, for $\mathrm{U}(N)$ gauge groups on orientable surfaces the theta term survives the continuum limit and it gives rise to a phase transition at $\theta=\pi$, similar to the ones observed in the $2 \mathrm{~d} \mathrm{U}(1)$ gauge model [3] and in $\mathrm{CP}(N)$ models at strong coupling [4].

The plan of the paper is as follows. In section 2 we calculate the partition function of $2 \mathrm{~d} \mathrm{U}(N)$ and $\mathrm{SU}(N)$ lattice Yang-Mills theories with a theta term using the standard group character ("strong coupling") expansion. In section 3 we derive the continuum limit of the action which will turn out to be the heat kernel with an additional contribution from the theta term. We interpret the continuum partition function in terms of the underlying topology and show the presence of a phase transition at $\theta=\pi$ in the topologically nontrivial cases. Recently it has been shown that 2d QCD is equivalent to a one dimensional gas of fermions [5]. In section 4 we discuss our results in this language. In particular we show that the topological term can be interpreted as an external magnetic field imposed on this fermion gas. Finally in section 5 we summarise our results and discuss their possible relevance to more realistic models with a topological term. 


\section{The partition function on the lattice}

In this section we derive an expression for the partition function of 2d Yang-Mills theories with a theta term. We use the standard strong coupling character expansion. The expression of the partition function in terms of the character expansion coefficients is well known on arbitrary surfaces, see e.g. [6, 7], so we shall just quote this result. We then calculate explicitly the character expansion coefficients for the specific lattice action that we pick.

We use the conventional Wilson lattice gauge model; the degrees of freedom are gauge group elements associated to links of a two dimensional square lattice. The gauge group $G$ can be thought of as either $\mathrm{SU}(N)$ or $\mathrm{U}(N)$ until some specific choice is stated. The action for a configuration $\left[U_{l}\right]$ is given by

$$
\mathcal{S}\left[U_{l}\right]=\sum_{p} s\left(U_{p}\right),
$$

where $U_{p}$ is the product of link variables around the plaquette $p, s$ is a conjugacy class function on $G$ (i.e. $s\left(g^{-1} U g=s(U), \forall g, U \in G\right)$ and the summation is for all the plaquettes. The partition function of a finite lattice consisting of $A$ plaquettes is

$$
Z_{A}(g)=\sum_{\left[U_{l}\right]} \exp \left(-\frac{N}{g^{2}} \sum_{p} s\left(U_{p}\right)\right),
$$

where $g$ is the gauge coupling and the summation is for all the configurations.

Since the Boltzmann factor on each plaquette is a conjugacy class function, it can be expanded in irreducible characters of $G$ as

$$
\exp \left(-\frac{N}{g^{2}} s(U)\right)=\sum_{r} d_{r} \Lambda_{r}\left(N / g^{2}\right) \chi_{r}(U),
$$

where

$$
\Lambda_{r}\left(N / g^{2}\right)=\frac{1}{d_{r}} \int_{G} d U \exp \left(-\frac{N}{g^{2}} s(U)\right) \chi_{r}^{*}(U) .
$$

Here $r$ labels the irreducible representations of $G, d_{r}$ is the dimension and $\chi_{r}$ is the character of $r$. Substituting (3) into the expression of the partition function, the link 
variables can be integrated out using the orthogonality of the characters. For a closed orientable surface of genus $g$ we obtain [6]

$$
Z_{A}(g)=\sum_{r} d_{r}^{2-2 g} \Lambda_{r}^{A}\left(N / g^{2}\right)
$$

For non-orientable surfaces the summation is restricted to self-conjugate representations and also there is an additional constant factor [7], which will be unimportant here, so we omit it.

Up to this point we have not specified the form of the action, (5) holds for any gauge invariant action. The information specific to the action is entirely contained in the $\Lambda_{r}$ 's. We now choose for the Yang-Mills part the usual Wilson action

$$
s_{w}(U)=\frac{1}{2}\left(\operatorname{tr} U+\operatorname{tr} U^{\dagger}\right)
$$

To make the analytic calculations feasible we choose

$$
s_{\theta}(U)=\frac{1}{2}\left(\operatorname{tr} U-\operatorname{tr} U^{\dagger}\right)
$$

for the theta term. Obviously this have the desired continuum behaviour which can be easily seen in exactly the same way as the continuum limit of the Wilson term. While in the continuum limit $s_{w}(U)$ is proportional to the square of the field strength (up to a trivial additive constant), the term $s_{\theta}(U)$ goes linearly with the field strength. Notice also that the Euclidean theta term is purely imaginary as it should be. With these notations we parametrise the one plaquette action as

$$
s(U)=-\cosh \epsilon s_{w}(U)+\sinh \epsilon s_{\theta}(U)
$$

where $\epsilon$ is a real parameter that determines the relative weight of the two terms. This parametrisation of the action was chosen for convenience. If we wanted the relative weight of the theta term to be the larger one, the convenient choice would be to exhange the cosh and the sinh. The calculation would go in essentially the same way.

In the reaminder of this section we explicitly calculate the integrals (四) with this action in both the $\mathrm{U}(N)$ and the $\mathrm{SU}(N)$ case. 
The integrand of (四) is a conjugacy class function so we can rewrite it in terms of the eigenvalues of $U,\left\{\exp \left(i \alpha_{1}\right), \exp \left(i \alpha_{2}\right), \ldots, \exp \left(i \alpha_{N}\right)\right\}$. In terms of these variables the action is

$$
s(\vec{\alpha})=-\sum_{j=1}^{N}\left(\cosh \epsilon \cos \alpha_{j}-i \sinh \epsilon \sin \alpha_{j}\right)
$$

The irreducible representations of $U(N)$ can be labelled by $N$ integers $\left\{n_{1} \geq n_{2} \geq, \ldots, \geq\right.$ $\left.n_{N}\right\}=[n]$. According to Weyl's character formula the character of this representation reads

$$
\chi_{[n]}(\vec{\alpha})=\frac{\operatorname{det}_{j k} \mathrm{e}^{i \alpha_{j}\left(n_{k}+N-k\right)}}{\Delta(\vec{\alpha})},
$$

where

$$
\Delta(\vec{\alpha})=\operatorname{det}_{j k} \mathrm{e}^{i \alpha_{j}(N-k)} .
$$

Finally the invariant measure on $U(N)$ is given by

$$
d U \propto \prod_{m=1}^{N} \frac{d \alpha_{m}}{2 \pi}|\Delta(\vec{\alpha})|^{2},
$$

up to a factor independent of the $\alpha$ 's. Putting this all together and introducing

$$
f(\alpha)=\frac{N}{g^{2}}(\cosh \epsilon \cos \alpha-i \sinh \epsilon \sin \alpha)
$$

as a shorthand notation we obtain

$$
\begin{gathered}
d_{[n]} \Lambda_{[n]}\left(N / g^{2}\right)=\text { const. } \prod_{l=1}^{N} \int_{-\pi}^{\pi} \frac{d \alpha_{l}}{2 \pi} \mathrm{e}^{f\left(\alpha_{l}\right)} \Delta(\vec{\alpha})\left(\operatorname{det}_{j k} \mathrm{e}^{i \alpha_{j}\left(n_{k}+N-k\right)}\right)^{*} \\
=\text { const. } \prod_{l=1}^{N} \int_{-\pi}^{\pi} \frac{d \alpha}{2 \pi} \mathrm{e}^{f\left(\alpha_{l}\right)}\left[\sum_{\sigma \in S_{N}}(-1)^{\sigma} \prod_{j=1}^{N} \mathrm{e}^{i \alpha_{j}\left(N-\sigma_{j}\right)}\right] \\
\times\left[\sum_{\eta \in S_{N}}(-1)^{\eta} \prod_{m=1}^{N} \mathrm{e}^{-i \alpha_{m}\left(n_{\eta(m)}+N-\eta(m)\right)}\right] \\
=\text { const. } \sum_{\mu \in S_{N}}(-1)^{\mu} \sum_{\sigma \in S_{N}} \prod_{l=1}^{N} \int_{-\pi}^{\pi} \frac{d \alpha_{l}}{2 \pi} \exp \left[f\left(\alpha_{l}\right)-i \alpha_{l}\left(\sigma(l)+n_{\mu \sigma(l)}-\mu \sigma(l)\right)\right],
\end{gathered}
$$

where the summations are on all the permutations of $N$ elements. The terms of the last sum on $\sigma$ are easily seen to be independent of $\sigma$ thus

$$
d_{[n]} \Lambda_{[n]}\left(N / g^{2}\right)=\text { const. } N ! \operatorname{det}\left[\int_{-\pi}^{\pi} \frac{d \alpha}{2 \pi} \mathrm{e}^{f(\alpha)-i \alpha\left(n_{j}+k-j\right)}\right]
$$


Now all that remains to be done is to calculate the Fourier expansion of $\mathrm{e}^{f(\alpha)}$

$$
\begin{aligned}
& \int_{-\pi}^{\pi} \frac{d \alpha}{2 \pi} \exp \left[\frac{N}{g^{2}}(\sinh \epsilon \cos \alpha-i \cosh \epsilon \sin \alpha)-i m \alpha\right] \\
= & \int_{-\pi}^{\pi} \frac{d \alpha}{2 \pi} \exp \left[\frac{N}{g^{2}} \cos (\alpha+i \epsilon)-i m \alpha\right]=\mathrm{e}^{-m \epsilon} I_{m}\left(N / g^{2}\right) .
\end{aligned}
$$

Here $I_{m}$ is the modified Bessel function of order $m$. Substituting this into (15) we obtain the final expression for the $\mathrm{U}(N)$ character expansion coefficients

$$
\Lambda_{[n]}^{U(N)}(g)=\frac{1}{d_{[n]}} \operatorname{det}_{j k}\left(\mathrm{e}^{-\epsilon\left(n_{j}-j+k\right)} I_{n_{j}-j+k}\left(N / g^{2}\right)\right)
$$

In the $S U(N)$ case there are two differences compared to $\mathrm{U}(N)$. At first the irreducible representations of $S U(N)$ are labelled by non-negative integers satisfying $n_{1} \geq n_{2} \geq, \ldots \geq n_{N}=0$, which does not change anything in the previous calculation. Secondly for $\mathrm{SU}(N)$ there is an additional $\sum_{k=1}^{N} \alpha_{k}=0$ constraint on the eigenvalues that must be enforced by a delta function in the measure. If this delta function is written as

$$
\delta\left(\sum_{k=1}^{N} \alpha_{k}\right)=\sum_{m=-\infty}^{\infty} \mathrm{e}^{i m \sum_{k=1}^{N} \alpha_{k}},
$$

the $U(N)$ calculation goes through without any modification giving the final result

$$
\Lambda_{[n]}^{S U(N)}\left(N / g^{2}\right)=\frac{1}{d_{[n]}} \sum_{m=-\infty}^{\infty} \operatorname{det}_{j k}\left[\mathrm{e}^{-\epsilon\left(n_{j}-j+k+m\right)} I_{n_{j}-j+k+m}\left(N / g^{2}\right) .\right]
$$

In the $\epsilon \rightarrow 0$ limit the action is pure Wilson and the expressions we obtained give the correct character expansion of the Wilson action. Had we chosen to give a bigger relative weight to the theta term by exchanging the cosh and the sinh, we would have gotten the same result but with Bessel functions instead of modified Bessel functions.

\section{The continuum limit}

In this section we calculate the continuum limit of the character expansion coefficients to leading (nontrivial) order in $g \rightarrow 0$. In 2 d gauge theories the convergence radius 
of the strong coupling expansion is infinite and it makes sense to calculate the weak coupling limit of the terms in a strong coupling expansion.

As $g \rightarrow 0$ we want the coupling of the theta term to approach its continuum value $\theta / 2 \pi$. Accordingly $\epsilon$ has to be scaled in this limit as

$$
\epsilon \propto \frac{\theta}{2 \pi} \frac{g^{2}}{N}
$$

This can also be seen from dimensional analysis. For our purpose the only "interesting" part of the $\Lambda_{[n]}$ 's is the one that depends on the representation label. From now on we drop representation independent factors and whenever we do so the " $\approx$ " sign will be used. Later on we shall comment on the information that might have been lost due to these omittions.

Substituting the asymptotic representation of the modified Bessel functions

$$
I_{n}(z) \longrightarrow \frac{e^{z}}{\sqrt{2 \pi z}} \mathrm{e}^{-\frac{n^{2}}{2 z}} \quad \text { as } \quad z \rightarrow \infty
$$

and (20) for $\epsilon$ into (17), for small $g$ we obtain

$$
\begin{aligned}
& \Lambda_{[n]}(g, \theta) \approx \frac{1}{d_{[n]}} \operatorname{det}_{j k} \mathrm{e}^{-\frac{g^{2}}{2 N}\left[\left(n_{j}-j+k\right)^{2}-\frac{\theta}{\pi}\left(n_{j}-j+k\right)\right]} \\
& \approx \frac{1}{d_{[n]}} \exp \left(-\frac{g^{2}}{2 N} \sum_{j=1}^{N} n_{j}\left(n_{j}-2 j+2-\frac{\theta}{\pi}\right)\right) \operatorname{det}_{j k} \mathrm{e}^{-\frac{g^{2}}{N}\left(n_{j}-j\right)(k-1)}
\end{aligned}
$$

In the last step the determinant was expanded as a sum over permutations, a factor, independent of the permutations was pulled out and the remainder was rewritten as a determinant. The last factor in (22) is a Van der Monde determinant which can be expressed as

$$
\operatorname{det}_{j k} \mathrm{e}^{-\frac{g^{2}}{N}\left(n_{j}-j\right)(k-1)}=\prod_{j<k}\left(\mathrm{e}^{-\frac{g^{2}}{N}\left(n_{j}-j\right)}-\mathrm{e}^{-\frac{g^{2}}{N}\left(n_{k}-k\right)}\right) .
$$

The last ingredient that we have not given yet is the dimension of the representation $[n]$,

$$
d_{[n]} \approx \prod_{j<k}\left(n_{k}-k-n_{j}+j\right) .
$$

Making use of this and the asymptotic expansion

$$
\ln \left(\frac{e^{-a x}-e^{-b x}}{b-a}\right) \longrightarrow \ln x-\frac{a+b}{2} x+\ldots \quad \text { as } \quad x \rightarrow 0
$$


one then obtains

$$
\frac{1}{d_{[n]}} \operatorname{det}_{j k} \mathrm{e}^{-\frac{g^{2}}{N}\left(n_{j}-j\right)(k-1)} \approx \exp \left(-\frac{g^{2}}{N}(N-1) \sum_{j=1}^{N} n_{j}\right)
$$

This completes the calculation of $\Lambda_{[n]}^{U(N)}$ in the continuum limit, giving

$$
\Lambda_{[n]}^{U(N)}(g, \theta) \approx \exp \left(-\frac{g^{2}}{2 N} \sum_{j=1}^{N} n_{j}\left(n_{j}-2 j+N+1-\frac{\theta}{\pi}\right)\right) .
$$

In the exponent for $\theta=0$ we recover the eigenvalue of the quadratic Casimir which gives the heat kernel action.

The previous calculation goes through in the $\mathrm{SU}(N)$ case without any modification, except that we have to do a little bit more work to evaluate the infinite sum in (19).

$$
\begin{aligned}
\Lambda_{[n]}^{S U(N)}(g, \theta) & \approx \sum_{m=-\infty}^{\infty} \exp -\frac{g^{2}}{2 N} \sum_{j=1}^{N}\left(n_{j}+m\right)\left(n_{j}+m-2 j+N+1-\frac{\theta}{\pi}\right) \\
& =\Lambda_{[n]}^{U(N)} \sum_{m=-\infty}^{\infty} \exp -\frac{g^{2}}{2 N}\left(N m^{2}+2 m \sum_{j=1}^{N}\left(n_{j}-\frac{\theta}{2 \pi}\right)\right) .
\end{aligned}
$$

In the $g \rightarrow 0$ limit this sum can be explicitly evaluated by approximating it with a Gaussian integral. After calculating this integral we obtain

$$
\begin{aligned}
\Lambda_{[n]}^{S U(N)} & \approx \exp -\frac{g^{2}}{2 N}\left[\sum_{j=1}^{N} n_{j}\left(n_{j}-2 j+N+1-\frac{\theta}{\pi}\right)-\frac{1}{N}\left(\sum_{j=1}^{N} n_{j}+\frac{N \theta^{2}}{4 \pi^{2}}\right)^{2}\right] \\
& =\exp -\frac{g^{2}}{2 N}\left[\sum_{j=1}^{N} n_{j}\left(n_{j}-2 j+N+1\right)-\frac{\left(\sum_{j=1}^{N} n_{j}\right)^{2}}{N}-N \frac{\theta^{2}}{4 \pi^{2}}\right]
\end{aligned}
$$

Again the $\theta=0$ case agrees with the character expansion of the heat kernel action.

Notice that while in the $\mathrm{U}(N)$ case $\theta$ couples to the representations through their diagonal $\mathrm{U}(1)$ charge $\sum_{j=1}^{N} n_{j}$, in the $\mathrm{SU}(N)$ case $\theta$ appears only in a representation independent term that can as well be dropped. This is exactly what we expected. Had we formulated the model in the continuum from the outset, the theta term would have been identically zero for $\mathrm{SU}(N)$. The reason for this is that the continuum topological term is proportional to the integral of the trace of the field strength and the trace vanishes for $\mathrm{SU}(N)$. 
Let us now take a closer look at the $\mathrm{U}(N)$ case. In the previous section we made a remark that on non-orientable surfaces only self conjugate representations contribute to the partition sum. In terms of the $n_{j}$ 's the condition for a representation to be self conjugate is

$$
n_{j}=-n_{N-j+1} \quad \forall j: 1 \leq j \leq N
$$

It follows that for self conjugate representations $\sum_{j=1}^{N} n_{j}=0$ and $\theta$ cannot be seen in the continuum at all. Recall that in this case the topological charge must be identically zero because the second cohomology group of any compact closed non-orientable $2 \mathrm{~d}$ manifold is trivial. Thus the integral of any closed 2-form and in particular that of the curvature must be zero. Indeed we do not expect the theta term to influence the continuum physics.

Finally let us deal with the only case in which there is a nontrivial topological term in the continuum, i.e. the gauge group $\mathrm{U}(N)$ on a compact closed orientable surface. In this case the configurations of the system are labelled by the irreducible representations of $\mathrm{U}(N)$, there is no additional constraint. The action corresponding to the representation $[n]$ is given by

$$
\frac{A g^{2}}{2 N} \sum_{j=1}^{N} n_{j}\left(n_{j}-2 j+N+1-\frac{\theta}{\pi}\right)+(2 g-2) \ln d_{[n]},
$$

where $A$ is the number of plaquettes forming the surface. In the thermodynamic limit when $A \rightarrow \infty$ the last term becomes irrelevant and the partition sum is completely dominated by the ground state, the representation that minimises

$$
s_{\theta}[n]=\sum_{j=1}^{N} n_{j}\left(n_{j}-2 j+N+1-\frac{\theta}{\pi}\right)
$$

If $\theta$ is varied, the ground state might change at some point(s) due to level crossing, which causes phase transitions. This phenomenon has already been observed in a variety of similar systems; $2 \mathrm{~d} U(1)$ gauge theory with a theta term [2, 3], the analogous 1d spin model [8] and in 2d $C P(N)$ models at strong coupling [4].

Let us now explore this possibility in the present model. For $\theta=0$ the action is

$$
s_{0}[n]=\sum_{j=1}^{N} n_{j}\left(n_{j}-2 j+N+1\right)=\sum_{j=1}^{N} n_{j}^{2}+\sum_{j=1}^{N} n_{j}(N+1-2 j) \geq \sum_{j=1}^{N} n_{j}^{2}
$$


The inequality can be easily verified by pairing the terms in the previous sum as

$$
\sum_{k=1}^{N / 2}\left[n_{k}(N+1-2 k)-n_{N-k+1}(N+1-2 k)\right] \geq 0
$$

and using the inequalities between the $n_{k}$ 's. If $N$ is odd then $k$ goes only to $\frac{N-1}{2}$ and the remaining term vanishes. It is clear from (33) that at $\theta=0$ the ground state is given by the trivial representation $n_{1}=\ldots=n_{N}=0$. This is not surprising since it is well known that in the strong coupling expansion of the Wilson action the trivial representation gives the largest contribution for any value of the gauge coupling [9].

Also from (33) we can see that

$$
s_{\theta}[n] \geq \sum_{j=1}^{N} n_{j}\left(n_{j}-\frac{\theta}{\pi}\right)=\sum_{j=1}^{N}\left(n_{j}-\frac{\theta}{2 \pi}\right)^{2}+\text { const. }
$$

which shows that by increasing $\theta$ starting from zero we do not encounter any phase transition up to $\theta=\pi$. At this point however there is a phase transition; above $\pi$ the representation $n_{1}=\ldots=n_{N}=1$ becomes the ground state. Due to the periodicity in $\theta$ the same type of phase transition occurs whenever $\theta$ becomes an odd integer times $\pi$. The free energy density around the $\theta=\pi$ phase transition is given by

$$
\begin{array}{rc}
f(\theta)=0 & 0 \leq \theta \leq \pi \\
f(\theta)=g^{2}\left(1-\frac{\theta}{\pi}\right) & \theta \geq \pi
\end{array}
$$

We have to note that (36) gives only the part of the free energy that depends on the representation explicitly. The reason for this is that throughout the calculation we kept only the representation dependent terms of the free energy. Any representation independent but theta dependent neglected terms, however, should be analytic at the phase transition. Thus in the $\mathrm{SU}(N)$ case, for example, we eventually would have needed to include an extra $\theta$-dependent renormalisation counter-term in the free energy to make it $\theta$-independent, as we expect it to be in the continuum. 


\section{The fermionic picture}

It is well known that $2 \mathrm{~d} \mathrm{U}(N)$ QCD is esentially equivalent to a system of $N$ fermions living on a circle [5]. It turns out that the result we obtained in the previous section has a nice interpretation in this language. The theta term produces an external magnetic field proportional to $\theta$ and the phase transition we encountered at $\theta=\pi$ corresponds to an abrupt change of the ground state of the fermion system. This correspondance is possibly not very surprising since the theta term and the external magnetic field represent the same ambiguity in the quantisation of the two equivalent systems. This ambiguity is due to the non-simply connected nature of the classical configuration space.

In this section we briefly summarise the fermion interpretation of $2 \mathrm{~d}$ QCD from our point of view and discuss the relevance of the theta term. For a discussion of the fermionic picture starting from a continuum Hamiltonian formulation we refer the reader to [5].

In section 2 we obtained the partition function of $2 \mathrm{~d}$ QCD using the Lagrangian description. Now we want to rewrite this in terms of the Hamiltonian in order to identify the (Euclidean) quantum mechanical system to which this partition function corresponds. In the Hamiltonian language the two coordinates on the surface correspond to time and one space direction. In order to simplify matters we first choose the simplest nontrivial surface, the cylinder. We take the compactified dimension to be "space" and time is measured along the axis of the cylinder. Using the gluing procedure described in section 2 and the form of the character expansion coefficients obtained in section 3 we can construct the partition function on a cylinder with arbitrarily chosen holonomies $U_{1}, U_{2} \in U(N)$ on the boundary

$$
Z_{L T}\left(g, U_{1}, U_{2}\right)=\sum_{[n]} \mathrm{e}^{-\frac{L T g^{2}}{2 N} s_{\theta}[n]} \chi_{[n]}\left(U_{1}\right) \chi_{[n]}^{*}\left(U_{2}\right),
$$

where $L$ and $T$ are the size of the cylinder in the space and time direction respectively. In the language of quantum mechanics the partition function of a system with fixed 
configurations at $t=0$ and $t=T$ is the (Euclidean) propagator

$$
\left\langle q_{2} T \mid q_{1} 0\right\rangle=\sum_{j}\left\langle q_{2} \mid j\right\rangle \mathrm{e}^{-E_{j} T}\left\langle j \mid q_{1}\right\rangle
$$

where the $|j\rangle$ 's form a complete set of eigenstates of the Hamiltonian and the $E_{j}$ 's are the corresponding energies. Comparing (37) and (38) we conclude that in 2d QCD the energy eigenstates are labelled by the irreducible representations and the corresponding eigenfunctions are the characters.

In this formulation the wave function is a function on the gauge group which might suggest that the classical configuration space is the group itself. This is however not quite right because the characters do not form a complete set in the space $\mathrm{L}^{2}(U(N))$, they span only the subspace of conjugation invariant functions on $\mathrm{U}(N)$. If we want to interpret the partition function of the cylinder as the propagator of a quantum mechanical system, the wave functions have to be restricted to be invariant under conjugation. This restriction looks more natural from the gauge theory point of view. At any fixed time $t$ the only (independent) gauge invariant observable is the conjugacy class of the holonomy around the "space" circle at $t$. It follows that the classical configuration space is not the whole gauge group but only the set of its conjugacy classes. The most convenient parametrisation of this set is given by the phases of the eigenvalues of the group elements as described in section 2. In this way the classical configuration space in the $\mathrm{U}(N)$ case can be identified with the $N$-torus $T^{N}$ (a maximal torus in $\mathrm{U}(N))$ factorised by the permutation group of $N$ elements. This factorisation is essential because all the permutations of a given set of eigenvalues correspond to the same conjugacy class. The classical configuration space is thus equivalent to that of a system of $N$ indistinguishable particles on a circle and the wave functions are symmetric functions on $T^{N}$.

There is however one more complication due to the nontrivial nature of the integration measure defining the inner product on the Hilbert space. Recall that the invariant measure in terms of the above given coordinate system is given by (12). The inner 
product is

$$
\langle\Psi \mid \Phi\rangle=\prod_{m=1}^{N} \int_{-\pi}^{\pi} \frac{d \alpha_{j}}{2 \pi} \Psi^{*}(\vec{\alpha}) \Delta^{*}(\vec{\alpha}) \Delta(\vec{\alpha}) \Psi(\vec{\alpha}),
$$

where $\Delta(\vec{\alpha})$ is the determinant (11). It is now seen that by including the determinant $\Delta$ in the wave function the inner product is written in terms of the "usual" measure on $T^{N}$. Since the original wave functions $\Psi$ were symmetric the redefined wave functions $\Psi \Delta$ will be antisymmetric. This shows that the particles we are dealing with are fermions.

To see that it is actually the whole Hilbert space of $N$ fermions that enters the expression for the propagator we have to look at the irreducible characters after the above redefinition

$$
\chi_{[n]}(\vec{\alpha}) \Delta(\vec{\alpha})=\operatorname{det}_{j k} \mathrm{e}^{i \alpha_{j}\left(n_{k}+N-k\right)}=\mathrm{e}^{i \frac{N-1}{2} \sum_{j=1}^{N} \alpha_{j}} \operatorname{det}_{j k} \mathrm{e}^{i \alpha_{j}\left(n_{k}+\frac{N+1}{2}-k\right)}
$$

and verify that they span the whole Hilbert space. (40) is essentially a Slater determinant composed of one-particle momentum eigenstates $\mathrm{e}^{i \alpha_{j} p_{k}}$ with momenta

$$
p_{k}=n_{k}+\frac{N+1}{2}-k \text {. }
$$

For $\mathrm{N}$ odd it is easily checked that the irreducible representations are indeed in oneto-one correspondance with the states in the $N$-fermion Fock space on a unit circle. If $N$ is even the allowed momenta are half integers which give antiperiodic boundary conditions for the $N$-fermion wave function.

The wave function (40) describes an energy eigenstate with energy

$$
E[p]=\frac{L g^{2}}{2 N} s_{\theta}[n]=\frac{L g^{2}}{2 N}\left[\sum_{j=1}^{N}\left(p_{j}-\frac{\theta}{2 \pi}\right)^{2}-\sum_{j=1}^{N}\left(\frac{N+1}{2}-j-\frac{\theta}{2 \pi}\right)^{2}\right]
$$

The first term is the energy of $N$ noninteracting particles in the presence of a magnetic field with constant $\theta / 2 \pi$ vector potential along the circle on which the particles live. The second term can be thought of as a trivial - though $\theta$-dependent - shift of the energy that does not depend on the state of the system. For $\theta<\pi$ the ground state is the usual fermionic ground state with all the states filled below the Fermi 
surface. The Fermi surface in this case degenerates to the two points $\pm p_{F}= \pm \frac{N-1}{2}$. In summary, we have succeded in showing that $2 \mathrm{~d} \mathrm{U}(N)$ QCD is equivalent to a system of $N$ noninteracting fermions moving on a unit circle. The correspondance between the amplitudes in the fermionic model and the gauge model is

$$
\langle\vec{\beta} T \mid \vec{\alpha} 0\rangle_{\text {fermion }}=\Delta^{*}(\vec{\beta}) \Delta(\vec{\alpha})\langle U(\vec{\beta}) T \mid U(\vec{\alpha}) 0\rangle_{\text {gauge }}
$$

In this language the partition function on the torus can be obtained by taking periodic boundary condition in the time direction and integrating out the holonomy around the two identified boundary pieces

$$
Z_{\text {torus }}(g)=\sum_{[p]} \mathrm{e}^{-T E[p]}
$$

This is the partition function of the corresponding fermion gas at temperature $1 / T$. In the zero temperature limit only the ground state contributes to (44). At $\theta=\pi$ however the ground state becomes degenerate and above $\pi$ the new ground state will be the one with the momenta of all the particles shifted by one unit. This is the phase transition that we encountered in the previous section.

Equation (37) gives the propagator in terms of momentum eigenstates. As a final check of our results it is instructive to rewrite the $N$-fermion propagator using position eigenstates. This can be done by using the Poisson summation formula

$$
\sum_{p=-\infty}^{\infty} f(p)=\sum_{m=-\infty}^{\infty} \int_{-\infty}^{\infty} d x f(x) \mathrm{e}^{2 \pi i m x}
$$

The constraint $p_{1}>p_{2} \ldots$ on the momenta can be dropped at the expense of a factor $N$ ! and we also omit the representation-independent term in $E[p]$. Using the notation $\Delta_{[p]}$ for the Slater determinant the fermion propagator reads

$$
\begin{aligned}
& \langle\vec{\beta} T \mid \vec{\alpha} 0\rangle \mathrm{e}^{i \frac{N-1}{2} \sum_{j=1}^{N}\left(\alpha_{j}-\beta_{j}\right)}=\sum_{p_{j}=-\infty}^{\infty} \Delta_{[p]}^{*}(\vec{\beta}) \mathrm{e}^{-\frac{L T g^{2}}{2 N} \sum_{j=1}^{N}\left(p_{j}-\frac{\theta}{2 \pi}\right)^{2}} \Delta_{[p]}(\vec{\alpha}) \\
= & \frac{1}{N !} \sum_{m_{j}=-\infty}^{\infty} \sum_{\sigma, \eta \in S_{N}}(-1)^{\sigma \eta} \prod_{j=1}^{N}\left[\int_{-\infty}^{\infty} d y_{j} \mathrm{e}^{-\frac{T L g^{2}}{2 N} y_{j}^{2}+i y_{j}\left(2 \pi m_{j}+\alpha_{\sigma(j)}-\beta_{\eta(j)}+\frac{i T L g^{2} \theta}{2 N \pi}\right)}\right]
\end{aligned}
$$




$$
\begin{aligned}
=\frac{1}{N !}\left(\frac{2 N \pi}{T L g^{2}}\right)^{\frac{N}{2}} & \sum_{m_{j}=-\infty}^{\infty} \mathrm{e}^{-\frac{i \theta}{2 \pi} \sum_{j=1}^{N}\left(2 \pi m_{j}+\alpha_{j}-\beta_{j}\right)} \\
& \times \sum_{\sigma, \eta \in S_{N}}(-1)^{\sigma \eta} \exp \left[-\frac{N}{2 T L g^{2}} \sum_{j=1}^{N}\left(2 \pi m_{j}+\alpha_{\sigma(j)}-\beta_{\eta(j)}\right)^{2}\right]
\end{aligned}
$$

This is the familiar form of the Euclidean propagator for a system of $N$ fermions on a circle. Each term in the sum over $m$-configurations corresponds to the contribution of paths with a particular set of winding numbers $\left[m_{1}, m_{2}, \ldots, m_{N}\right]$. In complete agreement with our expectation the theta term gives an extra phase factor to each winding sector.

Recall that if the configuration space of the classical system is not simlply connected quantisation becomes ambiguous. Paths belonging to different homotopy classes can get extra relative phase factors that belong to a representation of the first homotopy group of the classical configuration space. The ambiguity arises because there is no a priori criterium to select a particular representation [10]. The $\theta$-dependent phase factor in (46) represents exactly this quantisation ambiguity, the representations of the homotopy group being labelled by $\theta$. This concludes our discussion of the fermion gas interpretation of $2 \mathrm{~d} \mathrm{U}(N) \mathrm{QCD}$.

\section{Conclusions}

In this paper we have shown a way of incorporating a theta term in two dimensional $\mathrm{U}(N)$ and $\mathrm{SU}(N)$ gauge theories. Starting from a lattice formulation we derived the continuum limit of the action resulting in a generalisation of the heat kernel action in the presence of a topological term. Our choice of the lattice theta term was the most naive, the only requirement it satisfied was having the topological term (first Chern number) as its naive continuum limit. In this way the lattice "topological charge" was not necessarily an integer and it was not necessarily zero in the topologically trivial cases. In spite of this, in the continuum limit we have been able to recover the topological properties of the system. In the topologically trivial cases when the topological charge was expected to vanish identically $(\mathrm{SU}(N)$ gauge group on any 
surface and $\mathrm{U}(N)$ gauge group on non-orientable surfaces) the only effect of the theta term remaining in the continuum limit was a trivial constatant shift of the ground state energy. On the other hand in the topologically nontrivial case $(\mathrm{U}(N)$ gauge group on orientable surfaces) the theta term remained to be coupled to the dynamics in the continuum, giving rise to phase transitions at $\theta_{c}= \pm \pi, \pm 3 \pi, \pm 5 \pi \ldots$ The same type of phase transition has been observed in $\mathrm{CP}(N)$ models at strong coupling [4]. It is not very surprising that in the $2 \mathrm{~d}$ gauge system even in the weak coupling limit we obtain an essentially strongly coupled system. This is due to the fact that in $2 \mathrm{~d}$ QCD the strong coupling expansion has an infinite radius of convergence and we expect the strong coupling behaviour to persist down to any nonzero value of the coupling.

Recently, based on Monte Carlo calculations, Schierholz argued that in the two dimensional $\mathrm{CP}(3)$ model and in four dimensional SU(3) QCD this phase transition also takes place but the critical value of $\theta$ becomes small for weak coupling, eventually reaching zero in the continuum limit [1]. In this way $\theta$ is forced to be zero if confinement is to be maintained and this gives a solution to the strong $\mathrm{CP}$ problem. From the argument given at the end of the previous paragraph it is clear that our results for $2 \mathrm{~d}$ QCD give no direct hint concerning the phase transition in $2 \mathrm{~d} \mathrm{CP}(N)$ or $4 \mathrm{~d}$ QCD. Such a weak coupling transition must be directly related to the mechanisms responsible for maintaining confinement at arbitrarily weak coupling in four dimensions. We hope to address this question elsewhere.

In the previous section we interpreted the continuum theta term in the fermionic formulation of $2 \mathrm{~d} \mathrm{U}(N)$ QCD. In particular we have shown that in the fermion language the theta term corresponds to an external magnetic field imposed on the fermion gas. Very recently the connection between the gauge model an the fermion gas has been exploited to obtain some new results concerning the Calogero-Sutherland model [11]. Our results might also find some application in this direction. 


\section{Acknowledgements}

One of the authors (T.G.K.) is grateful to J. Wheater for introducing him to this problem. This research was supported partly by NSF Grant \# PHY-92-18990 and OTKA (Hungarian Research Fund) \# F14276.

\section{References}

[1] G. Schierholz, Theta vacua, confinement and the continuum limit, preprint DESY94-229 and hep-lat/9412083; G. Schierholz, Nucl. Phys. Proc. Suppl. 37A (1994) 203; S. Olejnik and G. Schierholz, Nucl. Phys. B Proc. Suppl. 34 (1994) 709

[2] U.J. Wiese, Nucl. Phys. B318 (1989) 153 and references teherein

[3] T.G. Kovács and J.F. Wheater, Mod. Phys. Lett. A6 No. 30 (1991) 2827; A.S. Hassan, M. Imachi and H. Yoneyama, Real space renormalization group analysis of $\mathrm{U}(1)$ gauge theory with a theta term in two dimensions, preprint KYUSHUHET-20 and hep-lat/9410003

[4] N. Seiberg Phys. Rev. Lett. 53 (1984) 637

[5] J.A. Minahan and A.P. Polychronakos, Nucl. Phys. B422 (1994) 172; M. Caselle, A. D'Adda, L. Magnena and S. Panzeri, Nucl. Phys. B416 (1994) 751

[6] B.Ye. Rusakov, Mod. Phys. Lett. A9 No. 9 (1990) 693;

[7] E. Witten, Commun. Math. Phys. 141 (1991) 153

[8] M. Asorey, J.G. Estevez and J. Salas, J. Phys. A Math. Gen. 27 (1994) 3707

[9] J-M. Drouffe, J-B.. Zuber, Phys. Rep. 102 (1983) 104

[10] L.S. Schulman, Techniques and applications of path integration, N.Y. Wiley, 1981 
[11] J.A. Minahan, A.P. Polychronakos, Density correlation functions in CalogeroSutherland models, preprint hep-th/9404192 